Journal of Computer Science 5 (12): 1095-1100, 2009

ISSN 1549-3636

(C) 2009 Science Publications

\title{
Unequally Error Protected Wireless Data Transmission Using Channel State Information and Adaptive Encoders
}

\author{
Yaseen H. Tahir, Chee Kyun Ng, N.K. Noordin, B.M. Ali and S. Khatun \\ Department of Computer and Communication Systems Engineering, \\ Faculty of Engineering, University Putra Malaysia, UPM, 43400 Serdang, \\ Selangor Darul Ehsan, Malaysia
}

\begin{abstract}
Problem statement: Higher data transmission rate with Quality of Services (QoS) guarantee is an important objective for wireless broadband communication systems. Unlike other communication system, real-time transmission requires high data rate and good error performance to ensure good quality of service. This study describes the design problem of real-time wireless data transmission which provides higher achievable data rate with Unequal Error Protection (UEP). Approach: Real-time data stream was divided equally into two streams, High Priority (HP) and Low Priority (LP) streams depending on their requirements. We considered these two bit streams as being encoded separately by two adaptive encoders. Feedback of Channel State Information (CSI) was used in adaptive physical layer channel for efficient use of the current available bandwidth by controlling the encoders. High code rate were used in both encoders when the channel is good while low code rate is otherwise. The two coded data streams were superimposed together with two different adjustable levels of power to achieve the UEP at the receiver end. Results: In this proposed scheme, same design metrics, namely, time, bandwidth and power were used to increase the transmission efficiency. Conclusion/Recommendations: Results showed that our scheme provided higher error performance for HP stream compared with other schemes that transmit using one power level data stream. The finding can be modified for studying the effects of fading on this system in future research.
\end{abstract}

Key words: Real-time wireless transmission, data rate, QOS, UEP, CSI, error performance

\section{INTRODUCTION}

Efficiently transmitting higher data rate for error-sensitive applications over error prone wireless channels is the main challenge in future wireless broadband communication systems. Exploiting channel states to transmit highest possible data rate is in great demand for many applications. Unequal Error Protection (UEP) is an effective method for data transmission in error prone environments. It provides different levels of protection to different parts of the data which have unequal degrees of importance. The requirements of Quality of Service (QoS) differ from one application to another. For example in the case of video conferencing, people always focus more on the participants rather than the background. Thus, the area of interest (in this case, the participants) should be allocated more resources, so that the received quality would be better than the area that is not of interest. Using UEP, the distribution of errors can be changed without incurring extra resource ${ }^{[1]}$.

In this study, a proposed scheme of UEP with adaptive data rate is given by exploiting the features of superposition coding and using adaptive encoders. Source data has to be divided into two bit streams with different priorities. The High Priority (HP) is for the bits stream which carries the important data while Low Priority (LP) is for the bits stream that carries less important data. Unlike LP data, we need to keep the errors in the HP data as low as possible because it has detrimental effects on the reconstructed data quality. Feedback of Channel State Information (CSI) for the current channel characteristics can give more moderate resources allocation since there is good information about the available channel bandwidth ${ }^{[2]}$.

Corresponding Author: Yaseen H. Tahir, Department of Computer and Communication Systems Engineering, Faculty of Engineering, University Putra Malaysia, UPM, 43400 Serdang, Selangor Darul Ehsan, Malaysia H/P:0060173370274 


\section{J. Computer Sci., 5 (12): 1095-1100, 2009}

$\operatorname{In}^{[3]}$, a solution given is based on Region Of Interest (ROI) by hierarchically partitioning the transmit packets in order to keep an acceptable QoS in the case of degraded conditions. The resource allocation strategy in terms of scheduling policy is between best-effort and guaranteed bit rate transmissions. $\operatorname{In}^{[4]}$ a sub-channel partitioning based on Unequal Error Protection (UEP) scheme for a Space-Time Block Coded Orthogonal Frequency Division Multiplexing (STBC-OFDM) system is proposed. At the receiver side of this system, OFDM sub-channels are partitioned into High-Quality (HQ) and Low Quality (LQ) groups according to the estimated channel qualities. Based on the feedback of sub-channels partitioning result, the transmitter assigns HP and LP video data to the corresponding HQ and LQ sub-channels. $\operatorname{In}^{[5]}$ real time video data is divided into two regions which are the ROI and the Remainder Region (RM). The two regions will be encoded such that the video transmission is adaptive to the current channel state, which is characterized by the effective data rate. Channel state parameters are fed back to the source coder to adjust the compression ratio of both regions.

In a real time communication system, the information about the time-varying channel characteristics can be used in transmission stage and is often available at the receiver and can be used during the decoding process. In general, based on the feedback of the CSI, suitable codes are selected to achieve higher data rate transmission with the required level of UEP. By exploiting this feedback, two different code rates are used in adaptive encoders to have adaptive data transmission. Since information and redundant bits are normally concatenated, most of each codeword bits are allocated for the information bits and the rest for the redundant bits in the case of good channel. In case of noisy channel, less information bits are assigned in the transmitted code words. Our aim is to provide higher data transmission with highest possible protection to achieve the best error performance.

Unequal error protection with CSI: Channel State Information (CSI) for the current channel characteristics is used for achieving a more proper resource allocation. In practical implementations, the channel state information must be estimated at the receiver and provided to the transmitter through a feedback channel ${ }^{[6]}$. It forms part of the signal model in wireless communications. The system usually needs to have some information regarding current channel characteristics to figure out what was sent from the transmitter or to enhance system performance, such as increasing transmission speed or setting degree of the UEP. The information can be the current channel characteristics, or the covariance of it. This type of information is generally referred as CSI and is usually estimated.

In order to achieve Unequal Error Protection (UEP) that discussed in ${ }^{[1,7,8]}$, the source data is to be split equally into two streams. One of these streams represents the HP data while the second one represents the LP data. Let $\mathrm{N}$ denotes the variance of Additive White Gaussian Noise (AWGN) channel with zero mean. Let $\mathrm{N}_{\mathrm{hp}}$ and $\mathrm{N}_{\mathrm{lp}}$ denote the prescribed variances of the Gaussian noise that the respective HP and LP bit streams need to withstand respectively, where it is naturally assuming that $\mathrm{N}_{\mathrm{hp}}>\mathrm{N}_{\mathrm{lp}}$. This means that bit streams can be decoded reliably with low levels of bit error rate when the channel noise level $\mathrm{N}$ is below than the prescribed noise levels. In the given proposed scheme, we have two different stages of UEP:

Firstly, the two bit streams will be encoded separately by two identical adaptive encoders and to be unequally protected against channel noise. To have UEP gain, the bit streams $\mathrm{xhp}$ and $\mathrm{x}_{\mathrm{lp}}$ are encoded with high channel code $\left(\mathrm{C}_{\mathrm{h}}\right)$ or low channel code $\left(\mathrm{C}_{1}\right)$ according to the CSI to generate high priority codeword $\left(\mathrm{v}_{\mathrm{hp}}\right)$ and high priority codeword $\left(\mathrm{v}_{\mathrm{lp}}\right)$ without changing system structure. For adaptive data rate transmission, $\mathrm{C}_{\mathrm{h}}$ which has higher code rate $\left(r_{h}\right)$ will be selected in the case of good channel allowing high number of information bits. In the case of bad channel state, $C_{1}$ with lower code rate $\left(r_{1}\right)$ will be selected where less information bits and more redundant bits are inserted.

Secondly, two signal constellations $S_{h p}$ and $S_{l p}$ for HP and LP streams respectively with different power levels are used for the second stage of UEP. The codewords $\mathrm{v}_{\mathrm{hp}}$ and $\mathrm{v}_{\mathrm{lp}}$ are mapped to the constellations and superimposed together before being transmitted as

$$
\mathrm{s}=\mathrm{s}_{\mathrm{hp}}+\mathrm{s}_{\mathrm{lp}}
$$

At the decoding end, the received sequence is:

$\mathrm{r}=\mathrm{s}+\mathrm{n}$

where, $\mathrm{n}$ is a sequence of independent and identically distributed (iid) Gaussian noise of variance N. If, $\mathrm{N}_{\mathrm{lp}}<\mathrm{N}<\mathrm{N}_{\mathrm{hp}}$, only the bit stream $\mathrm{x}_{\mathrm{hp}}$ is reliably decoded but if $\mathrm{N}<\mathrm{N}_{\mathrm{lp}}$, then both of the bit streams are reliably decoded. 


\section{J. Computer Sci., 5 (12): 1095-1100, 2009}

Superposition coding: In Superposition Coded Modulation (SCM), the different bit streams are transmitted on the same modulation intervals as shown by Bergmanns and Cover ${ }^{[7]}$. SCM scheme that uses block codes as the component code has been proposed in ${ }^{[9]}$. It has been shown that Superposition Block Coded Modulation (SBCM) can achieve a good gain with low encoding and decoding complexity with high UEP design flexibility. The rate design rule for SCM schemes with UEP requirements is proposed $\mathrm{in}^{[10]}$ by pointing the similarities between the SCM system and the multiple access channel. $\mathrm{In}^{[11]}$ systematic technique enabling reliable and energy efficient delivery of multiple scalable motion JPEG2000 video stream over a wireless local area network WLAN was introduced.

The superposition proposed scheme involves subtraction from the received signal of the estimated data for other transmitted streams, followed by decoding of the intended data stream ${ }^{[9-12]}$. Let:

$\alpha_{\mathrm{i}}=\mathrm{P}_{\mathrm{i}} / \mathrm{P}$

$\mathrm{P}=\mathrm{P}_{\mathrm{hp}}+\mathrm{P}_{\mathrm{lp}}$

where, $\alpha_{l}$ represents the fraction of the total allocation power $(\mathrm{P})$, while, $\mathrm{P}_{\mathrm{hp}}$ and $\mathrm{P}_{\mathrm{lp}}$ represent the power that assigned HP and LP streams respectively.

In this study, superposition coding is considered for UEP design with the condition that $\alpha_{\mathrm{hp}}>\alpha_{\mathrm{lp}}$ i.e., $\mathrm{P}_{\mathrm{hp}}>\mathrm{P}_{\mathrm{lp}}$.

System model: Scheme of adaptive channel transmission with UEP is depicted in Fig. 1 which consists of four major components; splitting circuit, adaptive encoding circuits, modulators and channel adaptive transmitter. The source data is split (equally in our assumption) into two streams, HP data stream and LP data stream according to their priorities. These two data streams are encoded separately by identical two-mode adaptive encoders. Feedback signal from channel adaptive transmitter is used to select encoder's mode of operation base on CSI. Lmode of $C_{1}$ and $r_{1}$ is selected when there is a bad channel state or no feedback signal but, if the channel is in good state, then $\mathrm{H}$-mode of $\mathrm{Ch}$ and $\mathrm{rh}$ is selected. Two Binary Phase Shift Keying (BPSK) modulators are used for both data streams with different power level. To achieve UEP, the total transmission power $\mathrm{P}$ is divided such that $0.8 \mathrm{P}$ is assigned to HP data and $0.2 \mathrm{P}$ to LP data. In this stage,

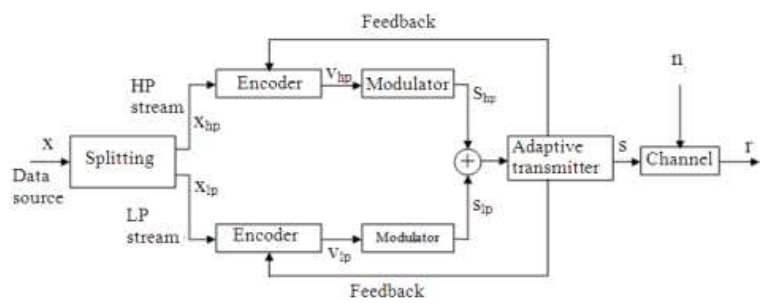

Fig. 1: Scheme of adaptive channel transmissions

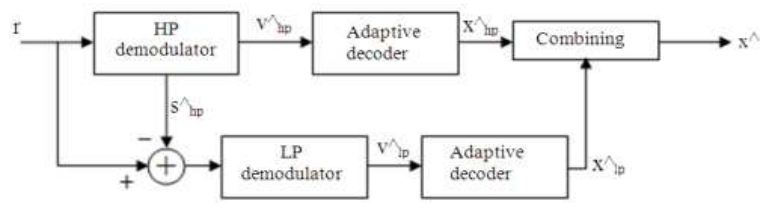

Fig. 2: Scheme of MSD receiver

UEP adjustment can be done by changing the power levels of the data streams depending on application requirements. Signals of both streams with different power levels are superimposed together in the summing node to produce the transmitted signal with adaptive data rate and UEP to exploit the available bandwidth.

Receiver design is shown in Fig. 2 were a MultiStage Decoding (MSD) procedure with adaptive decoding can be used. Decoders and encoders are assumed to be synchronized together in this system via CSI feedback. Since we have two data streams, then we have two-stages in MSD system to decode the transmitted information. In the first stage, it decodes the data of HP stream with most error protection $\hat{x}_{\mathrm{hp}}$, treating the signal from LP stream as a Gaussian interference. Once the receiver decodes the data of HP stream, it can reconstruct the signal of HP stream $\hat{s}_{\text {hp }}$ and subtract it from the received signal $r$. In the second stage the receiver can decode the data of LP stream $\hat{x}_{\mathrm{ip}}$ when only the background Gaussian noise is left in the system. The combining circuit is used to combine both of the decoded streams to reconstruct the transmitted data source.

Performance of proposed scheme: Consider application data source producing data stream with high quality requirements to be transmitted through AWGN channel. For higher spectral efficiency transmission, this data stream is split equally into two data streams according to their priorities. 


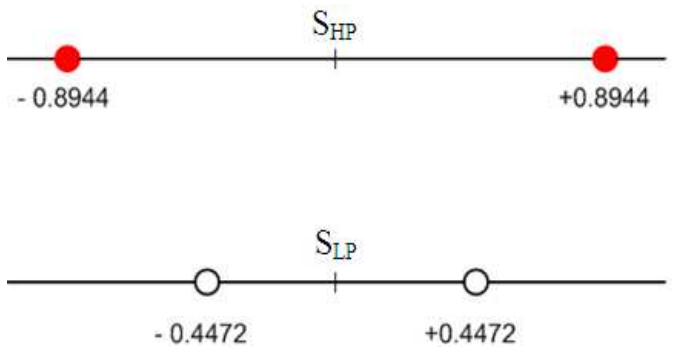

Fig. 3: Signal constellation of HP and LP data streams

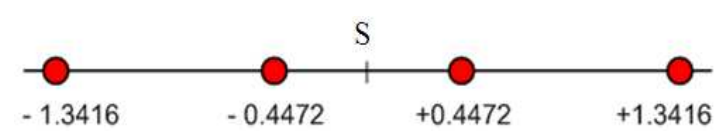

Fig. 4: Signal constellation of the transmitted signal

Without loss of generality, we use two simple block codes in our adaptive encoders. The modes of operation are; H-mode using a $(15,11)$ Hamming code $\mathrm{C}_{\mathrm{h}}$ with high code rate $\mathrm{r}_{\mathrm{h}}$ and L-mode using a $(7,4)$ Hamming code $C_{1}$ with low code rate $r_{1}$. The codewords $\mathrm{v}_{\mathrm{hp}}$ and $\mathrm{v}_{\mathrm{lp}}$ are modulated by BPSK modulators using $\mathrm{S}_{\mathrm{HP}}$ and $\mathrm{S}_{\mathrm{LP}}$ signal constellation, respectively. These signal constellations are shown in Fig. 3. The total average power is assumed to be 1 through this simulation. The transmitted signal $\mathrm{s}$ through $(0, \mathrm{~N})$ AWGN channel is a multilevel constellation as shown in Fig. 4.

At the receiving end, MSD procedure is used to demodulate and decode the received signal in two stages. First, HP data is demodulated and used in the second stage for LP data. The result of subtraction $\mathrm{s}^{\wedge}{ }_{\mathrm{hp}}$ signal from the received signal is demodulated and decoded by two-mode decoders depending on the CSI as well as the output of HP demodulator. The estimated data from both stages of decoding process is combined to generate the received data.

Simulation results showing the bit error rate of this scheme is given in Fig. 5 for $0.8 \mathrm{P}$ power assigned to HP stream and $0.2 \mathrm{P}$ assigned to LP stream. Curves of HP and LP data streams are shown and compared with the theoretical bit error rate of 4PAM for both encoders' modes of operation. It is observed that the performance of this system using $\mathrm{H}$ mode of operation is $0.6 \mathrm{~dB}$ less in average compared to traditional 4-PAM modulation when channel is good at a BER of $10^{-5}$.

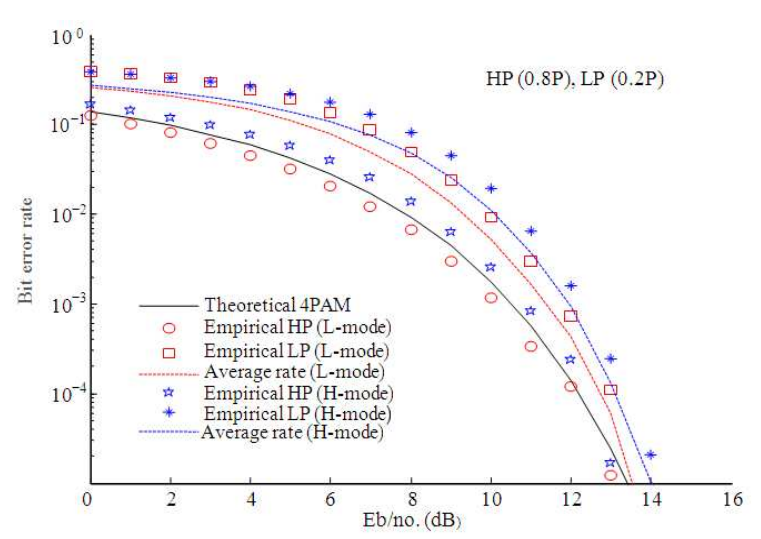

Fig. 5: BER comparison between empirical HP (0.8P) and LP (0.2P) data streams with the theoretical 4-PAM in both modes of operation $\mathrm{H}$-mode and L-mode

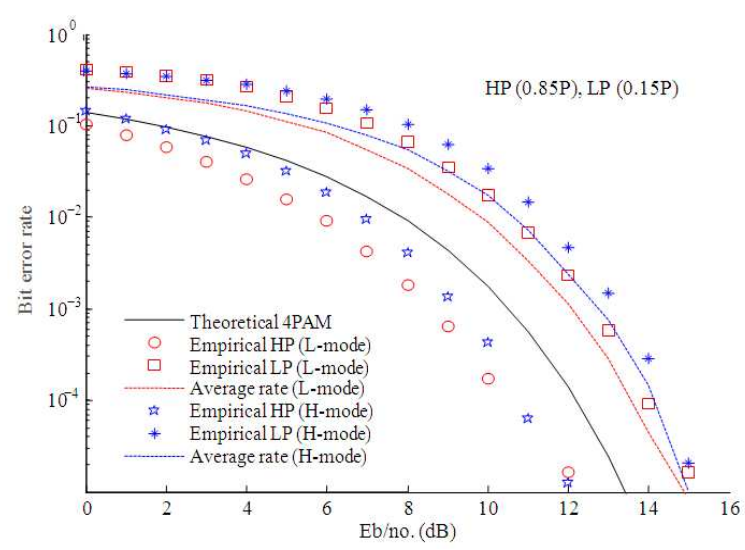

Fig. 6: BER comparison between empirical HP $(0.85 \mathrm{P})$ and LP $(0.15 \mathrm{P})$ data streams with the theoretical 4-PAM in both modes of operation $\mathrm{H}$-mode and L-mode

Less error protection for the LP data stream does not affect the performance of the receiving data while maintaining continuous reception with high data rate. In the case of losing feedback signal or bad channel condition, the system switch to L-mode of operation. It is obvious that the average performance is very close to 4-PAM modulation with $0.5 \mathrm{~dB}$ gain for the HP stream. From these curves in both modes of operation it is clear that we are achieving our target by transmitting the real time data by adaptive rate and UEP.

The BER performance results of the proposed scheme from both encoder modes with $0.85 \mathrm{P}$ power assigned to HP stream and $0.15 \mathrm{P}$ power to LP stream 


\section{J. Computer Sci., 5 (12): 1095-1100, 2009}

Table 1: Gain for HP stream in H-mode at $10^{-4}$ BER for three power distribution cases compared with the theoretical 4PAM

\begin{tabular}{lllll}
\hline $\begin{array}{l}\text { No. of } \\
\text { Figure }\end{array}$ & $\begin{array}{l}\text { Power distribution } \\
\mathrm{P}_{\mathrm{hp}}(\mathrm{w})\end{array}$ & $\mathrm{P}_{\mathrm{lp}}(\mathrm{w})$ & $\mathrm{P}_{\mathrm{hp}} / \mathrm{P}_{\mathrm{lp}}$ & $\begin{array}{l}\text { HP stream's } \\
\text { gain }(\mathrm{dB})\end{array}$ \\
\hline 5 & $0.8 * \mathrm{P}$ & $0.2 * \mathrm{P}$ & 4.00 & 0.5 \\
6 & $0.85 * \mathrm{P}$ & $0.15 * \mathrm{P}$ & 5.66 & 1.4 \\
7 & $0.75 * \mathrm{P}$ & $0.25 * \mathrm{P}$ & 3.00 & -1.8 \\
\hline
\end{tabular}

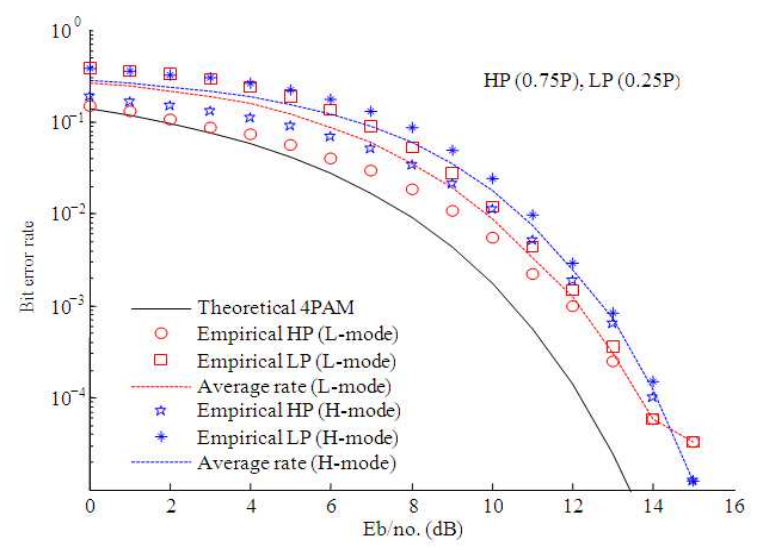

Fig. 7: BER comparison between empirical HP $(0.75 \mathrm{P})$ and LP $(0.25 \mathrm{P})$ data streams with the theoretical 4-PAM in both modes of operation $\mathrm{H}$-mode and L-mode

are compared with the theoretical BER of 4-PAM as shown in Fig. 6 It is shown from the results that by dividing the source stream into HP and LP streams according to their priority with high error protection level for HP stream, while less protection level is assigned to LP stream, much improved BER can be achieved for HP stream than the theoretical bit error rate of 4-PAM. There is about $1.5 \mathrm{~dB}$ gain for $\mathrm{HP}$ stream in $\mathrm{H}$-mode and little less for L-mode. This scheme is very useful in many applications that need more error protection performance for HP data stream.

The results of using $0.75 \mathrm{P}$ for HP stream and $0.25 \mathrm{P}$ for LP stream are depicted in Fig. 7. Figure 7 shows the effect of different power setting on BER performance for HP and LP streams in proposed scheme. By comparing these results with the results shown in Fig. 5 and 6, it can be seen that the proposed scheme give better performance when the difference between $P_{h p}$ and $P_{l p}$ fractions increase. This is due to our scheme gives batter performance at high UEP level as shown in Fig. 6. The gain for HP stream is increase at high UER level while it is decrease at low UEP level as shown in Table 1.
As mentioned earlier in unequal error protection with CSI Section, we have two stages of UEP in this proposed scheme. Using different power level distribution in stage 2 for the data streams gives flexibility for the desired error protection. However, as our proposed scheme achieves two enhancements in real-time wireless transmission compared with traditional techniques. The first improvement is keeping the HP stream in high protection against the error that may be accurse during the transmission process in error prone environments even in the case when the bad channel's condition presented. Unlike the traditional techniques, our results show another benefit such as continuity of transmission with adaptive transmission rate even if the channel was in bad state or no feedback signal from channel adaptive transmitter. This is done by switching to Lmode, rather than zero transmitted rate, with small deviation in BER performance.

\section{CONCLUSION}

High wireless data transmission in real time requirements with high error protection is a challenging target. This study has proposed a scheme for adaptive continuous real time transmission with two stages of different error protection levels. Channel state information, adaptive encoders and superposition coding are employed for this purpose. By splitting information data into two parts depending on their importance, the scheme focuses on how the encoders and superposition coding technique reacts with known CSI to achieve the preferable UEP. Based on the available channel bandwidth, HP and LP information data are sent with different power levels and code rates to manage continuous transmission with best quality. Adaptive transmission is achieved in this system by using the feedback of CSI to exploit the recourses (available power and bandwidth). System performance is compared with traditional techniques of transmission and shows good and flexible performance. Studying the effects of fading on this system is left for future research.

\section{REFERENCES}

1. Tahir, Y.H., W. Al-Hussaibi, C.K. Ng, N.K. Noordin and A. Al-Hemyari, 2008. Unequal error protection for wireless data transmission using superposition coding with feedback. Proceeding of the International Conference on Innovations in Information Technology, Dec. 1618, IEEE Xplore Press, USA., pp. 426-429. DOI: 10.1109/INNOVATIONS.2008.4781651 
2. Huang, C. and S. Liang, 2004. Unequal error protection for MPEG-2 video transmission over wireless channels. Signal Proc. Image Commun. J., 19: 67-79. http://www.ingentaconnect.com/content/els/0923 5965/2004/00000019/00000001/art00111

3. Nicolas T. and B. Pesquet-Popesc, 2007. Content based QoS differentiation for video streaming in wireless environment.

http://www.eurasip.di.uoa.gr/eurasip/Proceeding s/Eusipco/Eusipco2007/Papers/C3L-A04.pdf

4. Yang, G., D. Shen and O.K. Victor, 2004. UEP for video transmission in space-time coded OFDM system. Proceeding of the 23rd Annual Joint Conference on IEEE Computer and Communications Societies, Mar. 7-11, IEEE Xplore Press, USA., pp: 1200-1210. http://ieeexplore.ieee.org/xpl/freeabs_all.jsp?arn umber $=1357006$

5. Cover, T., 1972. Broadcast channels. IEEE Trans. Inform. Theor, IT-18: 2-14. http://ieeexplore.ieee.org/xpl/freeabs_all.jsp?arn umber $=1054727$

6. Geir, E., Henrik Holm and K.J. Hole, 2002. Adaptive coded modulation with imperfect channel state information: System design and performance analysis aspects. IEEE Int Symposium Advances in Wireless Communication, Sep. 2002, IEEE Xplore Press, USA., $\quad$ pp: 19-20. http://new.iet.ntnu.no/projects/beats/Documents/ ISWC2002.pdf

7. Bergmans, P. and T. Cover, 1974. Cooperative broadcasting. IEEE Trans. Inform. Theor., 20: 317-324.

http://ieeexplore.ieee.org/xpl/freeabs_all.jsp?arn umber $=1055232$
8. Cover, T.M., 1998. Comments on broadcast channels. IEEE Trans. Inform. Theor., 44: 2524-2530. DOI: $10.1109 / 18.720547$

9. Karabulut, G. and A. Yongacoglu, 2003. Superposition block coded modulation. Proceeding of the IEEE Canadian Conference on Electrical and Computer Engineering, May 4-7, IEEE Xplore Press, USA., pp: 1629-1632. DOI: 10.1109/CCECE.2003.1226220

10. Karabulut, G. and A. Yongacoglu, 2004. Rate design rule for superposition coded modulation. Proceeding of the IEEE Canadian Conference on Electrical and Computer Engineering, May 2-5, IEEE Xplore Press, USA., pp: 365-368. http://ieeexplore.ieee.org/xpl/freeabs_all.jsp?arn umber $=1345031$

11. Xin, J., P. Sofie and L. Gregory et al., 2006. Multi-user motion JPEG2000 over wireless LAN: Run-time performance-energy optimization with application-aware cross-layer scheduling. J. Zhej. Univ. Sci. A, 7: 151-158. DOI: 10.1631/jzus.2006.AS0151

12. Cronie, H.S., 2007. superposition coding for power and bandwidth efficient communication over the gaussian channel. Proceeding of the IEEE International Symposium on Information Theory, June 24-29, IEEE Xplore Press, USA., pp: 2311-2316. DOI: 10.1109/ISIT.2007.4557564 